\title{
The Effects of Varied Energy Source and Detergent on the Growth of Sterol Mutants of Saccharomyces cerevisiae
}

\author{
By NORMAN D. LEES, ${ }^{*}$ STEPHANIE L. LOFTON, ${ }^{1}$ ROBIN A. WOODS ${ }^{2}$ \\ AND MARTIN BARD ${ }^{1}$ \\ ${ }^{1}$ Department of Biology, Indiana University-Purdue University at Indianapolis, \\ Indianapolis, Indiana 46205, U.S.A. \\ ${ }^{2}$ Department of Biology, University of Winnipeg, Winnipeg, Canada $R 3 B 2 E 9$
}

(Received 29 August 1979; revised 20 November 1979)

\begin{abstract}
The effects of altered membrane sterol composition on the growth characteristics of sterol mutants of Saccharomyces cerevisiae were determined using various energy sources and the detergent Tergitol. These mutants do not synthesize the end-product sterol, ergosterol, but do not require exogenous sterol for growth. The sterol biosynthetic intermediates that are incorporated into the mutant membranes are related to alterations in growth characteristics. The C-24 transmethylation step (erg6) was shown to be critical to membrane integrity. Cells with this lesion were protected by the presence of Tergitol or glycerol in the medium. A double mutant (erg6/2), containing the $\Delta^{8} \rightarrow \Delta^{7}$ isomerization lesion (erg2) and the C-24 transmethylation lesion (erg6), was highly sensitive to ethanol and Tergitol. These results corroborate permeability and membrane fluidity studies indicating that $\mathrm{C}_{27}$ sterols are much less efficient than $\mathrm{C}_{28}$ sterols in maintaining normal membrane structure and function.
\end{abstract}

\section{INTRODUCTION}

Sterols have been identified as important components of biological membranes influencing critical membrane functions such as transport and the activity of membrane-bound enzymes (Demel \& de Kruyff, 1976; Cobon \& Haslam, 1973; de Kruyff et al., 1972). The precise manner in which sterols interact with proteins and other lipids within the membrane is not well understood. Electron spin resonance (e.s.r.) investigations using spin-labelled fatty acids which intercalate into the membrane have provided some information concerning these interactions (Berliner, 1976; Henry \& Keith, 1971; Rottem et al., 1970; Hsia et al., 1971). For example, in model membrane systems cholesterol was found to have a condensing effect on lipid bilayers in the liquid crystalline state while having a liquefying effect on bilayers in the gel or crystalline state (Hsia et al., 1971; Demel \& de Kruyff, 1976). Extensive studies in this area using biological membranes have been hampered by the fact that biological membranes are scrupulously regulated in their lipid content (de Kruyff, 1975). This makes sterol substitution and the adjustment of membrane sterol concentration impossible in most systems. Thus, studies have been restricted to membranes such as those from guinea-pig erythrocytes (Kores et al., 1972) and Mycoplasma mycoides (Rottem et al., 1970; Rottem et al., 1973) where variable sterol composition is possible.

Sterol mutants of Saccharomyces cerevisiae offer several advantages for evaluating the contribution of sterols to the structure and function of biological membranes. These mutants are unable to synthesize ergosterol, the end-product sterol, but require no exogenous sterol for growth. The altered sterol compositions of these mutants have been extensively characterized biochemically and genetically (Bard et al., 1977; Barton et al., 
Table 1. Presumptive enzyme defects and principal sterol intermediates accumulated in wild-type and sterol mutants of S. cerevisiae

$\begin{array}{ll}\text { Strain } & \text { Blocked biosynthetic step } \\ \text { A184D (wild-type) } & \\ \text { erg2 } & \Delta^{8} \rightarrow \Delta^{7} \text { isomerization } \\ \text { erg3 } & 5(6) \text { desaturation } \\ \text { erg5 } & 22(23) \text { desaturation } \\ \text { erg6 } & \text { C-24 transmethylation } \\ \text { erg6/2 } & \Delta^{8} \rightarrow \Delta^{7} \text { isomerization and } \\ & C-24 \text { transmethylation }\end{array}$

Sterol intermediates accumulated

Ergosterol (ergosta-5,7,22-trien-3 $\beta$-ol)

Zymosterol (cholesta-8,24-dien-3 $\beta$-ol)

Ergosta-8-en-3 $\beta$-ol

Ergosta-8,22-dien-3 $\beta$-ol

Ergosta-5,8,22-trien- $3 \beta$-ol

Ergosta-7,22-dien-3 $\beta$-ol

Ergosta-8,22-dien-3 $\beta$-ol

Ergosta-7,22,24(28)-trien-3 $\beta$-ol

Ergosta-8,22,24(28)-trien-3 $\beta$-ol

Ergosta-5,7-dien-3 $\beta$-ol

Zymosterol

Ergosta-7,24(28)-dien-3 $\beta$-ol

Zymosterol

Cholesta-5,7,22,24-tetraen-3 $\beta$-ol

Zymosterol

1974; Barton et al., 1975; Molzhan \& Woods, 1972). The enzymic step in ergosterol biosynthesis that is presumed blocked and the sterol intermediates that are accumulated and incorporated into the membranes of each strain have been determined (Table 1). Using e.s.r. techniques the substitution of sterol intermediates for ergosterol has been shown (Kleinhans et al., 1979) to alter radically the permeability of one sterol mutant to nickel. In addition, several sterol mutants have been shown to have altered permeability characteristics to various mono-, di- and trivalent cations and to crystal violet dye (Bard et al., 1978). Subsequent e.s.r. investigations have correlated substituted sterol intermediates with changes in membrane fluidity (Lees et al., 1979).

The mutants used in this study are of importance since they were originally selected on the basis of their resistance to the polyene antibiotic nystatin. Very little evidence has appeared concerning the physiological effects of altered sterol composition in this or other systems. One such report (Thompson \& Parks, 1974) indicated that a sterol mutant, nys3, of $S$. cerevisiae (allelic to the erg3 mutant in this report) showed decreased fermentative growth and greatly decreased growth on ethanol (aerobic growth). Another report (Pierce et al., 1978), using nystatin-resistant mutants of Candida albicans, indicated that the most highly resistant mutants had decreased growth rates and growth yields when compared with the wild-type strain. In this report differences in growth rate and growth yield among sterol mutants of $S$. cerevisiae grown on various energy sources and the effects of the non-ionic detergent Tergitol are reported.

\section{METHODS}

Organisms. The sterol mutants used in this investigation are derived from the wild-type strain of Saccharomyces cerevisiae, A184D, and have been described elsewhere (Bard et al., 1977; Barton et al., 1974; Barton et al., 1975; Molzhan \& Woods, 1972). Mutant erg2 is blocked at the $\Delta^{8} \rightarrow \Delta^{7}$ isomerization step, mutant erg 3 is blocked at the 5(6) desaturation step, mutant erg5 is unable to desaturate the 22(23) position, and mutant erg6 is unable to perform the C-24 transmethylation step in ergosterol biosynthesis (Table 1). Mutant erg6/2 is a double mutant obtained by genetically combining the single mutants erg6 and erg 2 into the same strain.

Media. Solid media consisted of $1 \%(\mathrm{w} / \mathrm{v})$ Difco yeast extract, $2 \%(\mathrm{w} / \mathrm{v})$ Bacto-peptone, $2 \%(\mathrm{w} / \mathrm{v})$ glucose and $2 \%(\mathrm{w} / \mathrm{v})$ agar (YEP/glucose agar). For some media, $2 \%(\mathrm{v} / \mathrm{v})$ ethanol $(\mathrm{YEP} / \mathrm{ethanol})$ or $3 \%(\mathrm{v} / \mathrm{v})$ glycerol (YEP/glycerol) was substituted for glucose; in others, energy sources were combined at the above concentrations. The non-ionic detergent Tergitol NP-40 (Sigma) was added at $1.5 \%(\mathrm{v} / \mathrm{v})$ where indicated.

Determination of mean generation times (m.g.t.). Cells grown aerobically overnight on YEP/glucose agar 
at $30^{\circ} \mathrm{C}$ were inoculated into YEP/glucose broth. After overnight growth at $30^{\circ} \mathrm{C}$ samples were removed and centrifuged $(5000 \mathrm{~g})$. The cells were washed once in the medium to be tested and suspended in $20 \mathrm{ml}$ of the same medium in a $250 \mathrm{ml}$ nephelometer flask. Growth at $30^{\circ} \mathrm{C}$ was monitored turbidimetrically using a Klett-Summerson colorimeter with a no. 66 red filter $(640$ to $700 \mathrm{~nm})$. Sample sizes were adjusted to yield an initial absorbance of 0.04 to 0.06 . Generation times were calculated from readings taken during the exponential phase (absorbance of $0 \cdot 1$ to $0 \cdot 3$ ). Growth of all liquid cultures was carried out in a rotary shaker.

Determination of growth yields. Cells were grown in an identical manner to that described for m.g.t. determination. The final suspension of washed cells was made in $250 \mathrm{ml}$ Erlenmeyer flasks containing $40 \mathrm{ml}$ of the medium to be tested. Sample sizes were adjusted to yield an initial absorbance of 0.04. The flasks were incubated at $30^{\circ} \mathrm{C}$ for $72 \mathrm{~h}$, and then the samples were harvested by centrifuging (5000 g) and dried for $48 \mathrm{~h}$ at $62^{\circ} \mathrm{C}$. The dry weights were determined for each sample.

\section{RESULTS AND DISCUSSION}

The results (Table 2) presented in this report indicate the relative physiological effects of various sterol substitutions on growth rate (mean generation time) and growth yield. Mutants erg 2 and erg5 grown on glucose (primarily fermentative growth) showed essentially no difference in rate or yield compared with the wild-type. Mutant erg3 had a slightly decreased growth rate but a similar growth yield to the wild-type. The significantly decreased growth rates of mutants erg6 and erg6/2 indicated that membranes which have primarily $\mathrm{C}_{27}$ sterols rather than $\mathrm{C}_{28}$ sterols (Table 1) are deficient in their ability to grow under these conditions. However, growth yields indicated that mutant erg6/2 was able to grow for a longer period than erg6 under these conditions.

A previous investigation (Parks et al., 1978) has indicated that many sterol mutants are also deficient in their ability to grow on non-fermentable carbon sources. Mutants erg6 and erg6/ 2 were unable to grow on ethanol while the other mutants and the wild-type showed greatly decreased growth rates but only slightly lower growth yields. This indicated that these strains could continue to grow on ethanol for an extended period of time. The ability to utilize glucose in the presence of ethanol was tested in order to establish whether the inability of mutants erg6 and erg6/2 to respire ethanol was due to respiratory deficiency or to sensitivity to ethanol, or to a combination of both factors. Fermentative growth in the presence of ethanol (YEP/glucose/ethanol) indicated that mutant erg6 was unaffected by ethanol, its growth rate and growth yield being equal to those shown on glucose alone. The growth rates and growth yields for the wild-type and mutants $\operatorname{erg} 2$ and $\operatorname{erg} 5$ also remained unchanged. Mutant erg6/2 showed a significantly lower growth rate and growth yield on YEP/glucose/ethanol, indicating a sensitivity to ethanol coupled with a decreased ability or inability to metabolize ethanol.

The effect of the non-ionic detergent Tergitol was tested since other types of yeast membrane mutants require detergent for growth or have noticeable increases or decreases in growth capacity in the presence of detergents (M. Bard, unpublished results). Tergitol was chosen since it would not serve as a metabolic substrate or a source of fatty acids. Thus, the effects here would be limited to stabilization or destabilization of membranes with varying sterol content. All the strains tested showed decreased growth yields in the presence of Tergitol, with the exception of mutant erg6 which showed a significantly increased growth rate and growth yield. The wild-type and mutant erg 2 showed no change in growth rate while the growth rates of the other three mutants were substantially decreased. The decreased growth yields noted for the wild-type and four of the mutants might have been due to a synergistic effect of Tergitol plus the ethanol that accumulated as the result of glucose fermentation. This appeared to be the case for mutant erg3 and the wild-type and, to a lesser extent, for mutants erg2 and erg6/2 since growth yields were less on YEP/glucose/ethanol/ Tergitol than on YEP/glucose/Tergitol or YEP/glucose/ethanol. Growth of mutant erg6 on YEP/glucose/ethanol/Tergitol was equal to that on YEP/glucose/Tergitol, again indicating that this strain is insensitive to ethanol and has a stabilized membrane structure in the presence of Tergitol. 


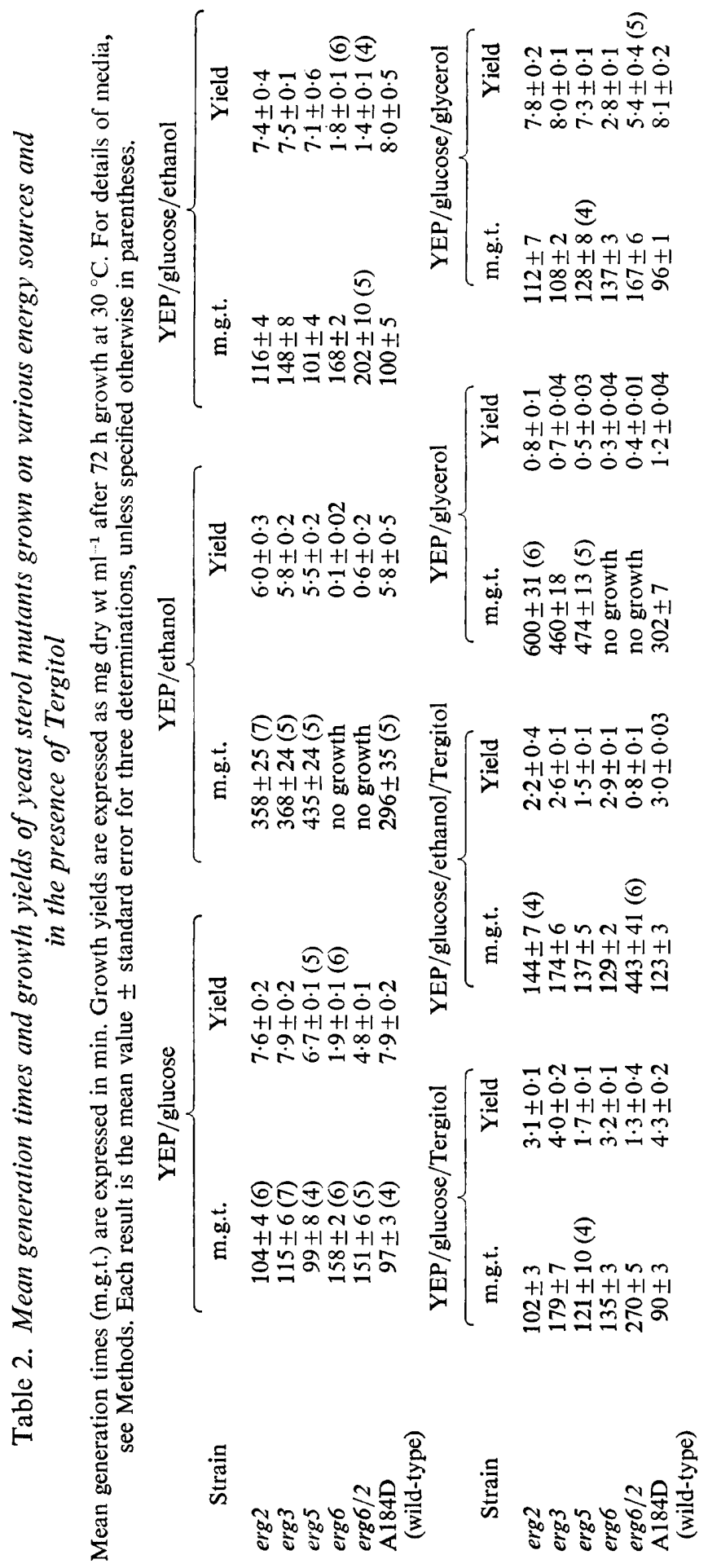


A second non-fermentable energy source which would not be expected to have an adverse effect on membrane structure or function was tested. Preliminary studies showed that mutants erg6 and erg6/2 could not grow on glycerol and the other strains had a greatly decreased capacity to utilize it. On glucose plus glycerol medium, the growth rates and yields for mutants erg2, erg3, erg6/2 and the wild-type were equal to those obtained on glucose medium. Mutant erg6 was protected by the glycerol in YEP/glucose/glycerol medium, showing an increased growth rate and growth yield. However, mutant erg 5 showed a decreased growth rate but the same yield as found with YEP/glucose. This result remains unexplained.

With the exception of an overall decrease in the ability to grow on non-fermentable energy sources and the adverse synergistic effects of ethanol plus Tergitol, mutant erg2 and the wild-type grew with media additives with the same efficiency as noted on YEP/glucose. Thus, the $\Delta^{8} \rightarrow \Delta^{7}$ isomerization block, with the concomitant accumulation of sterol intermediates (Table 1), had little effect upon the growth properties of this strain. The 5(6) desaturation lesion of mutant $\operatorname{erg} 3$ resulted in increased sensitivity to ethanol and Tergitol. Mutant erg5 showed decreased growth with Tergitol but was relatively unaffected by ethanol. The C-24 transmethylation step (erg6) was of much greater consequence in terms of membrane integrity as the growth rate of this mutant on glucose was severely reduced and it was unable to grow on non-fermentable energy sources. Ethanol had no adverse effect on the membranes of mutant erg6, while Tergitol and glycerol stabilized them permitting faster and more extensive growth. The combination of the C-24 transmethylation block and the $\Delta^{8} \rightarrow \Delta^{7}$ isomerization lesion in the same strain (mutant erg6/2) produced a synergistic effect. This strain showed increased sensitivity to ethanol and Tergitol individually and an extreme sensitivity to ethanol and Tergitol together. This increased sensitivity over that noted in mutant erg6 can be related to the accumulation of zymosterol as the principal membrane sterol rather than the combination of zymosterol and cholesta-5,7,22,24-tetraen-3 $\beta$-ol found in mutant erg6.

Previous results have shown that mutants erg6 and erg6/2 are more sensitive to cations and dyes than other sterol mutants (Bard et al., 1978) and that mutant erg6/2 is more permeable to nickel than wild-type yeast (Kleinhans et al., 1979). The present results corroborate the impaired membrane function indicated by these previous studies pointing out that the erg6 lesion and the combination of the erg6 and erg2 mutations are particularly disruptive of normal membrane function. Preliminary results have indicated that mutants erg6 and $\operatorname{erg} 6 / 2$ show $50 \%$ and $170 \%$ increases in doubling times, respectively, when grown on YEP/glucose at $35^{\circ} \mathrm{C}$. The wild-type and mutants erg2, erg 3 and erg 5 showed no changes in growth rate at $35^{\circ} \mathrm{C}$. This investigation also corroborates results previously obtained which indicated that some sterol mutants are reduced in their capacity to grow aerobically (Thompson \& Parks, 1974) and some sterol mutants show generally decreased growth rates (Pierce et al., 1978). In this study growth rates and sensitivity to various medium additives can be related to specific membrane sterol composition.

This investigation was supported in part by a PHS grant made to Indiana University under the Biochemical Research Support Grant Program (N.D.L.), a Purdue University XL Summer Faculty Fellowship (M. B.), and a Squibb Institute for Medical Research Grant, E. R. Squibb and Sons, Inc. (M. B.). We thank Michael Myers, David Ashmore and Alicia Garcia for their excellent technical assistance. 


\section{REFERENCES}

Bard, M., Woods, R. A., Barton, D. H. R., CorRIE, J. E. T. \& WidDowson, D. A. (1977). Sterol mutants of Saccharomyces cerevisiae: chromatographic analysis. Lipids 12, 645-654.

Bard, M., Lees, N. D., Burrows, L. S. \& KleiNHANS, F. W. (1978). Differences in crystal violet uptake and cation-induced death among yeast sterol mutants. Journal of Bacteriology 135, 1146-1148.

Barton, D. H. R., Corrie, J. E. T., Widdowson, D. A., BARD, M. \& Woods, R. A. (1974). Biosynthesis of terpenes and steroids. Part IX. The sterols of some mutant yeasts and their relationship to the biosynthesis of ergosterol. Journal of the Chemical Society, Perkin Transactions I, 1326-1333.

Barton, D. H. R., Gunatilaka, A. A. L., Jarman, T. R., Widdowson, D. A., BARD, M. \& Woods, R. A. (1975). Biosynthesis of terpenes and steroids. Part X. The sterols of some yeast mutants doubly defective in ergosterol biosynthesis. Journal of the Chemical Society, Perkin Transactions I, 88-92.

Berliner, L. J. (editor) (1976). Spin Labeling Theory and Applications, 1st edn. New York: Academic Press.

Cobon, G. S. \& Haslam, J. M. (1973). The effect of altered membrane sterol composition on the temperature dependence of yeast mitochondrial ATPase. Biochemical and Biophysical Research Communications 52, 320-326.

Demel, R. A. \& DE KRUYfF, B. (1976). The function of sterols in membranes. Biochimica et biophysica acta 457, 109-132.

Henry, S. A. \& Keith, A. D. (1971). Membrane properties of saturated fatty acid mutants of yeast revealed by spin labels. Chemistry and Physics of Lipids 7, 245-265.

Hsia, J. C., Schneider, H. S. \& Smith, I. C. P. (1971). A spin label study of the influence of cholesterol on phospholipid multibilayer structures. Canadian Journal of Biochemistry 49, 614-622.

Kleinhans, F. W., Lees, N. D., Bard, M., HaAK, R. A. \& Woods, R. A. (1979). ESR determinations of membrane permeability in a yeast sterol mutant. Chemistry and Physics of Lipids 23, 143154.
Kores, J., Oswald, R. \& KeIth, A. (1972). Erythrocyte membranes-compression of lipid phases by increased cholesterol content. Biochimica et biophysica acta 274, 71-74.

DE KRUYFF, B. (1975). Lipid-sterol interactions in liposomes and membranes. Biochemical Society Transactions 3, 618-621.

de Kruyff, B., Demel, R. A. \& van Deenen, L. L. M. (1972). The effect of cholesterol and epicholesterol incorporation on the permeability and on the phase transition of intact Acholeplasma laidlawii cell membranes and derived liposomes. Biochimica et biophysica acta 255, 331-347.

LeEs, N. D., Bard, M., Kemple, M. D., HAAK, R. A. \& Kleinhans, F. W. (1979). ESR determination of membrane order parameter in yeast sterol mutants. Biochimica et biophysica acta 553, 469-475.

Molzhan, S. W. \& Woods, R. A. (1972). Polyene resistance and the isolation of sterol mutants in Saccharomyces cerevisiae. Journal of General Microbiology 72, 339-348.

Parks, L. W., Mclean-Bowen, C., Taylor, F. R. \& Hough, S. (1978). Sterols in yeast subcellular fractions. Lipids 13, 730-735.

Pierce, A. M., Pierce, A. D., Jr, Unrau, A. M. \& Oehlschlager, A. C. (1978). Lipid composition and polyene antibiotic resistance of Candida albicans mutants. Canadian Journal of Biochemistry 56, 135-142.

Rottem, S., Húbbell, W. L., Hayflick, L. \& MCConNell, H. M. (1970). Motion of fatty acid spin labels in the plasma membrane of Mycoplasma. Biochimica et biophysica acta 219, 104-113.

Rottem, S., Yashoav, J., Neeman, Z. \& Razin, S. (1973). Cholesterol in Mycoplasma membranes. Composition, ultrastructure and biological properties of membranes from Mycoplasma mycoides var. capri cells adapted to grow with low cholesterol concentrations. Biochimica et biophysica acta 323, 495-508.

Thompson, E. D. \& Parks, L. W. (1974). Effects of altered sterol composition on growth characteristics of Saccharomyces cerevisiae. Journal of Bacteriology 120, 779-784. 